\title{
Comorbidities can predict the mortality of acute kidney injury requiring continuous renal replacement therapy: comparison with the Charlson comorbidity index
} \author{
Jae Yoon Park ${ }^{6}$

\footnotetext{
${ }^{1}$ Division of Nephrology, Department of Internal Medicine, Dongguk University College of Medicine, Gyeongju, Korea

${ }^{2}$ Department of Biostatistics, Dongguk University Ilsan Hospital, Goyang, Korea

${ }^{3}$ Division of Nephrology, Department of Internal Medicine, Seoul National University College of Medicine, Seoul, Korea

${ }^{4}$ Department of Internal Medicine, Yonsei University College of Medicine, Seoul, Korea

${ }^{5}$ Department of Internal Medicine, Seoul National University College of Medicine, Seoul, Korea

${ }^{6}$ Department of Internal Medicine, Dongguk University College of Medicine, Gyeongju, Korea
}

Jinwoo Lee ${ }^{1}$, Jiyun Jung ${ }^{2}$, Jangwook Lee ${ }^{3}$, Jung Tak Park ${ }^{4}$, Yong Chul Kim ${ }^{5}$, Dong Ki Kim ${ }^{5}$, Jung Pyo Lee ${ }^{5}$, Sung Jun Shin ${ }^{6}$,

Background: Comorbid conditions are important in the survival of patients with severe acute kidney injury (AKI) requiring continuous renal replacement therapy (CRRT). The weights assigned to comorbidities to predict survival may vary based on the type of index disease and advances in the management of comorbidities. We developed a modified Charlson comorbidity index $(\mathrm{mCCl})$ in patients with AKI requiring CRRT (mCCl-CRRT), thereby improving risk stratification for mortality.

Methods: A total of 1,583 patients received CRRT from 2008 to 2016 from two university hospital cohort were included to develop a comorbidity score. The weights of the comorbidities, per the $\mathrm{CCl}$, were recalibrated using a Cox proportional hazards model including age, sex, albumin, hemoglobin, and 15 types of $\mathrm{CCI}$ disease. The modified index was validated by 419 patients received CRRT from 2008 to 2016 from other two university hospital cohort. The c statistic of the area under curve as well as the net reclassification improvement values were confirmed in order to test the accuracy of the classification by $\mathrm{CCl}$ and $\mathrm{mCCl}-\mathrm{CRRT}$.

Results: A total of 1,583 participants were included in development cohort where average age was $62.04 \pm 14.29$ years, males were $970(61.3 \%), 777$ deaths (49\%) occurred, and average following days were $17.59 \pm 1.68$. The patients of $33 \%$ had cancer. The mCCI-CRRT showed no difference in c statistics (0.73) compared with the original $\mathrm{CCl}$, but improved net mortality risk reclassification by $25.27 \%$ ( $95 \%$ confidence interval, $0.0878-0.4176 ; \mathrm{P}=0.00267)$ relative to the original $\mathrm{CCl}$. When stratified by $\mathrm{CCl}$ and mCCI-CRRT score, the survival probability of CRRT patients was well-categorized according to the mCCI-CRRT score while CCI does not adequately classify the survival probability by score.

Conclusions: The mCCl-CRRT stratifies the risk for mortality in AKI patients who requiring CRRT better than the original $\mathrm{CCl}$, suggesting that it may be a preferred index for use in clinical practice.

Corresponding author: Jae Yoon Park

E-mail: nephrojyp@gmail.com

(C) The Korean Society for Transplantation

This is an Open Access article distributed under the terms of the Creative Commons Attribution Non-Commercial License (http://creativecommons.org/licenses/by-nc/4.0/) which permits unrestricted non-commercial use, distribution, and reproduction in any medium, provided the original work is properly cited. 